\title{
Path Antenna Gain in an Exponential Atmosphere
}

\author{
W. J. Hartman and R. E. Wilkerson
}

(May 7, 1959)

\begin{abstract}
The problem of determining path antenna gain is treated here in greater detail than previously $[1,2,3] .{ }^{1}$ The method used here takes into account for the first time the exponential decrease of the gradient of refractive index with height, and a scattering cross section inversely proportional to the fifth power of the scattering angle. Results are given for all combinations of beamwidths and path geometry, assuming that symmetrical beams are used on both ends of the path and that atmospheric turbulence is isotropic. The result appears as a function of both of the beamwidths, in addition to other parameters, and thus the loss in gain cannot be determined independently for the transmitting and receiving antennas. The values of the loss in gain are generally lower than the previous estimates for which a comparison is possible.
\end{abstract}

\section{Introduction}

Theories of the forward scatter of radio waves in the troposphere or stratosphere usually differ only in their assumptions about the radio wave scattering cross section and pertinent meteorological parameters. With any theory, the total effective gain of highly directive antennas is less over a scatter circuit than it would be in free space. The "path antenna gain" is less than or equal to the sum of the free space gains $G_{t}$ and $G_{r}$ of the transmitting and receiving antennas. Expressed in decibels above the gain of an isotropic radiation [1],

$$
G_{p} \leq G_{t}+G_{r} .
$$

Booker and de Bettencourt [2], Norton, Rice, and Vogler [1], and more recently Staras [3] have estimated the loss in antenna gain $G_{L}$, where $G_{L}$ in decibels is given by

$$
G_{L}=G_{t}+G_{r}-G_{p}
$$

for an atmosphere in which the variance of the gradient of the radio refractive index is either constant or varies parabolically with height. This paper estimates $G_{L}$ with a model atmosphere in which the refractive index decreases exponentially with height. $G_{L}$ is evaluated using antenna radiation patterns which are the same horizontally and vertically. To the authors' knowledge, $G_{L}$ has not been evaluated using antenna patterns which are not symmetric about the beam axis. Figures 6 and 7 show graphs of $G_{L}$ for various values of the parameters, and section 4 summarizes important definitions and gives a simplified method for predicting $G_{L}$. The reader who desires only an estimate of $G_{L}$ may skip to section 4 .

\section{Antenna Gain in Free Space}

The gain function $f(\theta, \phi)$ is the ratio of the power radiated in the direction $(\theta, \phi)$ per unit solid angle to the average power radiated per unit solid angle. This expresses the increase in power radiated in a given direction by the antenna over that from an isotropic radiator emitting the same total power. It is convenient to express the gain pattern of an antenna and its free space gain in terms of the electric field distribution $F(\xi, \eta)$ over the antenna aperture. Figure 1 shows a coordinate system with an antenna aperture in the $x, y$ plane and centered at the origin.

\footnotetext{
1 Figures in brackets indicate the literature references at the end of this paper.
} 


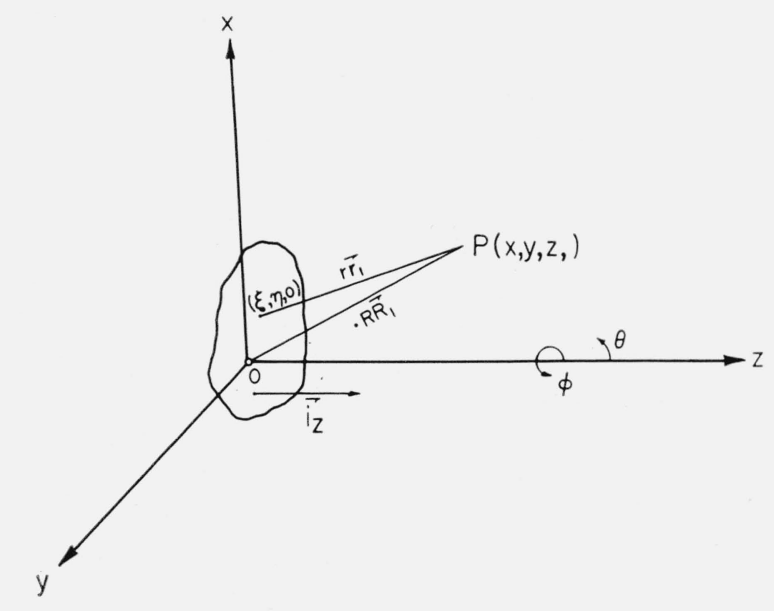

Figure 1. Geometry for determining the gain of an antenna.

The point $P(x, y, z)$ is in free space in the Fraunhofer or far zone. Using this notation, the power radiated per unit solid angle in a given direction by an antenna having constant phase distribution over the aperture is approximately [4]

$$
P(\theta, \phi)=\frac{\sqrt{\epsilon / \mu}}{2 \lambda^{2}}\left|\int_{A} F(\xi, \eta) \exp [j k \sin \theta(\xi \cos \phi+\eta \sin \phi)] d \xi d \eta\right|^{2},
$$

where $\sqrt{\mu / \epsilon}$ is the impedance of the propagating medium, $\lambda$ is the free space radio wavelength, $k=2 \pi / \lambda$, and $d \xi d \eta$ is a surface element of the aperture. The average power radiated per steradian in terms of the total power $P_{\mathrm{a}}$ across the aperture is

$$
P_{a} / 4 \pi=\frac{\sqrt{\epsilon / \mu}}{8 \pi} \int_{A}|F(\xi, \eta)|^{2} d \xi d \eta
$$

and the ratio of $(2-1)$ to $(2-2)$ is the gain function.

$$
f(\theta, \phi)=4 \pi P(\theta, \phi) / P_{a} .
$$

If the aperture illumination $F(\xi, \eta)$ is symmetrical about the origin, the maximum value $f_{\max }(\theta, \phi)$ of $(2-3)$ corresponds to the direction $\theta=0$ along the antenna beam axis, the $z$-axis in figure 1 ,

$$
f_{\max }(\theta, \phi)=f(0, \phi)=A_{e} /\left(\lambda^{2} / 4 \pi\right) .
$$

The factor $A_{e}$, called the "effective absorbing area" of an antenna, is given in terms of the distribution function as

$$
A_{e}=\left|\int_{A} F(\xi, \eta) d \xi d \eta\right|^{2} / \int_{A}|F(\xi, \eta)|^{2} d \xi d \eta
$$

For an isotropic radiator, $A_{e}=\lambda^{2} / 4 \pi$, as follows from (2-1) and (2-2). For any antenna with a uniformly illuminated aperture $(F(\xi, \eta)$ constant), the effective area equals the actual area. as may readily be seen from $(2-5)$. For the more usual $10 \mathrm{db}$ tapered illumination of the aperture of a parabolic dish antenna, the effective area $A_{e}$ is about 56 percent of the actual area.

The free-space gain of an antenna in decibels above the gain of an isotropic radiator is

$$
G=10 \log _{10} f_{\max }(\theta, \phi)=10 \log _{10}\left[A_{e} /\left(\lambda^{2} / 4 \pi\right)\right] \mathrm{db} .
$$

Define the normalized gain function, $g_{m}(\theta, \phi)$ :

$$
g_{m}(\theta, \phi)=f(\theta, \phi) / f_{\max }(\theta, \phi) .
$$


Figure 2 shows the normalized gain function $g_{m}(\theta, \phi)$ versus $\theta$ for a uniformly illuminated circular aperture of 100 wavelengths diameter. Figure 2 would be the same for any value of $\phi$. Figure 2 also shows a "square" antenna power pattern, where $g_{m}(\theta, 0)$ maintains the value unity for $0 \leq \theta \leq \delta$ and is zero when $\theta$ exceeds $\delta$.

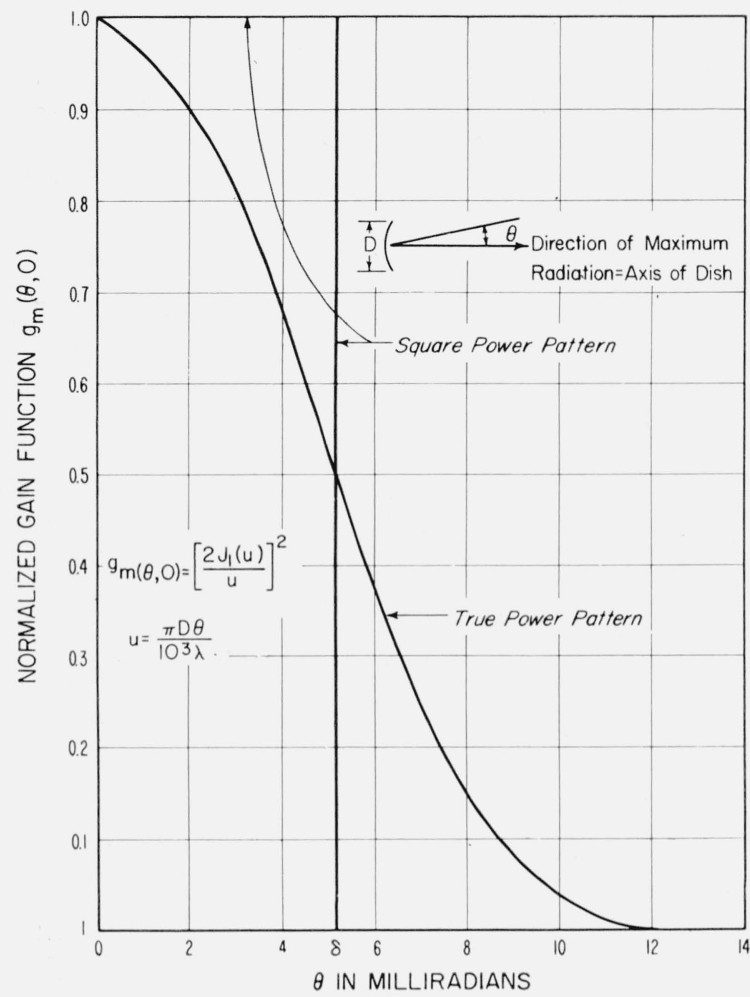

FIGURE 2. Computed polar pattern (without side lobes) for a parabolic dish (circular aperture uniformly illuminated) with $\mathrm{D} / \lambda=100$

The "square" power pattern will be used throughout the calculations in this paper.

For practical purposes the gain is often specified in terms of the half-power beamwidth, $2 \delta$. For an antenna with a symmetrical distribution of field intensity over the aperture one obtains [4]

$$
2 \delta \approx \lambda \sqrt{\mu_{0} / \mu_{2}},
$$

where $\mu_{i}$ is the $i$ th moment of the function $F(x)$ which gives the aperture field in terms of $x=\sqrt{\xi^{2}+\eta^{2}}$. Combining $(2-1),(2-7)$, and $(2-8)$.

or

$$
f(0, \phi) \approx\left(\mu_{0} / \mu_{2}\right) /(2 \delta)^{2}
$$

$$
f(0, \phi)=C_{1} /(2 \delta)^{2},
$$

where $C_{1}$ is a constant of proportionality depending on the characteristics of the antenna used. As examples, with the beamwidth $2 \delta$ expressed in radians, $C_{1}=7.2$ for a half-wave dipole, and $C_{1}=8.2$ for a parabolic dish with the edge illumination $10 \mathrm{db}$ down from the center illumination. This constant $C_{1}$ can be determined for some types of antennas using tables in the book, Reference Data for Radio Engineers [5].

The half-power beamwidth in figure 2 is $2 \delta$, where $\delta$ is the value of $\theta$ corresponding to $g_{m}(\theta)=$ 0.5 For the square pattern, assuming that $\delta$ is small, the power is radiated through $\pi \delta^{2}$ steradians if the aperture $A$ is circular, and through $(2 \delta)^{2}$ steradians if $A$ is square. The power gain $f(0, \phi)$ is then $4 \pi / \pi \delta^{2}$ for the circular aperture or $4 \pi /(2 \delta)^{2}$ for the square aperture, that is, $C_{1}=16$ or $C_{1}=4 \pi$. 
$G_{L}$, as determined by calculations in this report, depends directly on the semibeamwidths $\delta_{t}$ and $\delta_{r}$, and to a lesser extent on the shapes of the associated power patterns. Subscripts $t$ and $r$ in this paper refer to transmitting and receiving antennas, respectively.

\section{Scatter Integral}

If we denote by $K$ the received power relative to free space of a given scatter propagation circuit with arbitrary antennas, and by $K_{0}$ the received power relative to free space of the same circuit using isotropic antennas, then the loss in gain in decibels may be defined as

$$
G_{L}=-10 \log _{10} \frac{K}{K_{0}}
$$

and the total path antenna gain is

$$
G p=G_{t}+G_{r}-G_{L}
$$

The loss in gain $G_{L}$ depends on the plane wave power patterns of the two antennas and upon the path parameters in such a way that the effect of one antenna cannot be evaluated independently of the power pattern of the other.

In this report, it will be assumed that the antennas are sufficiently high so that ground reflection effectively doubles the power incident on the scattering volume over what it would be in free space and again doubles the power at the receiver. In general, $K$ is given by the volume integral

$$
K=d^{2} \int_{V} \frac{g_{t} g_{r} \sigma d v}{\left(R R_{0}\right)^{2}}
$$

where $d$ is the straight line distance between transmitter and receiver, $R$ and $R_{0}$ are distances from transmitter and receiver, respectively, to a scattering element $d v$, and $g_{t}$ and $g_{r}$ are $2 g_{m}$ and $2 g_{m r}$, that is, double the power patterns defined by $(2-7)$. The scattering cross section $\sigma$ gives the fractional part of the incident power scattered in a given direction (at an angle, $\alpha+\beta$ from the forward direction) per unit volume per unit solid angle. Refer to figure 3, which shows

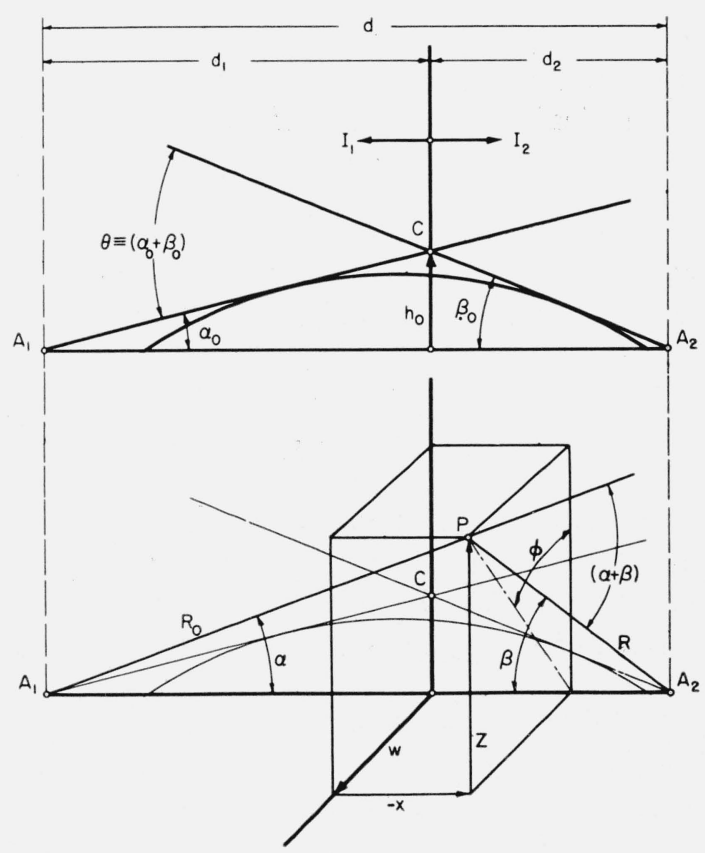

Figure 3. Geometry for the scattering integral. 
some of the geometry appropriate for the evaluation of (3-2). In this figure and throughout the remainder of this paper, $\theta$ is the angular distance, or the minimum scattering angle. A detailed discussion of the derivation of the form for $\sigma$ used here may be found in a recent paper [6]. The following result is obtained:

$$
\sigma=\frac{3 \lambda<(\Delta n)^{2}>}{4 \pi l_{0}^{2}}\left[2 \sin \left(\frac{\alpha+\beta}{2}\right)\right]^{-5}
$$

where $\alpha+\beta$ is the angle between the directions $R$ and $R_{0}$ as shown in figure $3, \lambda$ is the wavelength, $\left\langle(\Delta n)^{2}\right\rangle$ is the variance of refractive index fluctuations in the scattering medium, and $l_{0}$ is a "scale of turbulence" appropriate for the scattering medium, assuming homogeneous and isotropic turbulence. Following Booker and Gordon [7], Abbott [8] derived in a substantially different fashion the following expression for $\left\langle(\Delta n)^{2}\right\rangle / l_{0}^{2}$ :

$$
<(\Delta n)^{2}>/ l_{0}^{2}=\mathrm{S}^{\prime} \exp (-2 \gamma h)=S \exp (-2 \gamma z)
$$

where $S^{\prime}$ is the value of this parameter at sea level, $h \cong z-h_{0} / 2, S \cong S^{\prime} \exp \left(\gamma h_{0}\right)$, and $1 / \gamma$ is the scale height of the atmosphere, here assumed constant.

Using the coordinate system shown in figure 3, (3-2) becomes

$$
K=\frac{3 \lambda S d^{2}}{\pi} \int_{x_{1}}^{x_{2}} d x \int_{z_{1}}^{z_{2}} d z \int_{-\infty}^{\infty} d w \frac{g_{m t} g_{m r} \exp (-2 \gamma z)}{\left[2 \sin \left(\frac{\alpha+\beta}{2}\right)\right]^{5}\left(R R_{0}\right)^{2}} .
$$

Using the approximation

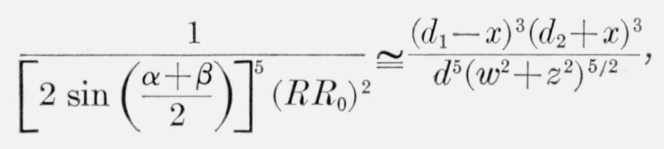

which is justified in appendix 1, and assuming that the effects of back scatter are negligible so that we may limit our $x$ integration to the region between the transmitter and receiver, and limiting $z$ to the region above the horizon rays, we obtain

$$
K=\frac{3 \lambda}{4 \pi d^{3}}\left\{\int_{0}^{d_{1}} d x \int_{z_{1 m}}^{\infty} d z \int_{-\infty}^{\infty} d w f(v)+\int_{-d_{2}}^{0} d x \int_{z_{2 m}}^{\infty} d z \int_{-\infty}^{\infty} d w f(v)\right\},
$$

where

$$
f(v)=g_{m t} g_{m r} \frac{\left(d_{1}-x\right)^{3}\left(d_{2}+x\right)^{3} S e^{-2 \gamma z}}{\left(w^{2}+z^{2}\right)^{5 / 2}}
$$

and

$$
\begin{aligned}
& z_{1 \mathrm{~m}} \simeq \beta_{0}\left(d_{2}+x\right) \\
& z_{2 \mathrm{~m}} \cong \alpha_{0}\left(d_{1}-x\right)
\end{aligned}
$$

We can express $K$ in terms of four parameters, $g_{m}, g_{m r}$,

$$
s=\frac{\alpha_{0}}{\beta_{0}}
$$

and

$$
\eta=\gamma d \theta
$$

where

$$
\theta=\alpha_{0}+\beta_{0},
$$

Thus

$$
K=K\left(s, \eta, g_{t}, g_{r}\right) .
$$


For isotropic antennas $g_{m t}=g_{m r}=1$, and thus $g_{t}=g_{r}=2$, including the effects of ground reflection as explained previously. $K$ then becomes

$$
\begin{aligned}
K(s, \eta, 2,2) & \equiv K_{0}=\frac{3 \lambda S}{\pi d^{3}}\left\{\int_{0}^{d_{1}} d x \int_{z_{1 \mathrm{~m}}}^{\infty} d z \int_{-\infty}^{\infty} d w \frac{\left(d_{1}-x\right)^{3}\left(d_{2}+x\right)^{3} e^{-2 \gamma z}}{\left(w^{2}+z^{2}\right)^{5 / 2}}\right. \\
& \left.+\int_{-d_{2}}^{0} d x \int_{z_{2 m}}^{\infty} d z \int_{-\infty}^{\infty} d w \frac{\left(d_{1}-x\right)^{3}\left(d_{2}+x\right)^{3} e^{-2 \gamma z}}{\left(w^{2}+z^{2}\right)^{5 / 2}}\right\}
\end{aligned}
$$

$(3-15)$ is evaluated exactly in appendix 2 with the result given by (7-14). $K(3-7)$ was evaluated by imposing geometric limits on the integrals and using Taylor series expansions. The limits vary as $s \mu<1, s \mu=1$, or $s \mu>1$ where

$$
\mu=\frac{\delta_{r}}{\delta_{t}}
$$

Figure 4 is a composite drawing and figure 5 is a drawing showing the $x w$ and $x h$ planes for $s \mu<1$. This leads to five integrals corresponding to the regions shown in figure 5 since it was found necessary to evaluate the integrals corresponding to the regions $I_{42}$ and $I_{43}$ as one integral, and then subtract the integral for $\mathrm{I}_{43}$. The integral $K$ was also evaluated numerically with $\left\langle(\Delta n)^{2}>/ l_{0}^{2}\right.$ proportional to $z^{-2}$ and $z^{-4}$, using the antenna power pattern $\left[2 J_{1}(u) / u\right]^{2}$ of figure 2 (without side lobes), and the value obtained for $G_{L}$ was found to be always within one and a half decibels of the value obtained using a "square" pattern with the same half-power beamwidth and height dependence.

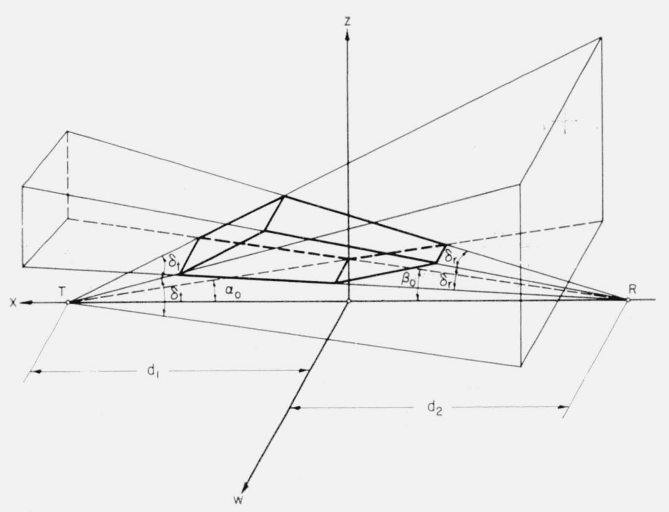

FIGURE 4. Scattering volume with "square" antenna patterns.

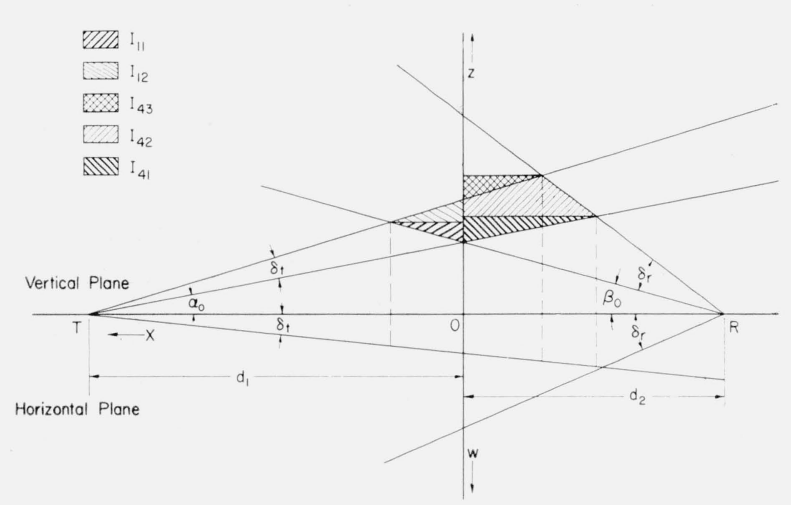

FIgURE 5. Shape of scattering volume in vertical and horizontal planes, $\mathrm{s} \mu>1$.

Effects of anisotropic turbulence have not been evaluated in this paper, but it can be shown that anisotropic turbulence arising from scale length changes has no effect on $K_{0}$ [isotropic antennas] except to add a multiplicative factor of $r$, where

$$
r=\frac{l_{v}}{l_{w}}=\frac{\text { scale of turbulence in vertical dimension }}{\text { scale of turbulence in horizontal dimension }} \text {. }
$$

However, it has been shown $[6,9]$ that anisotropic turbulence produces the following effect in the integrand, (3-8) of the scatter integral (3-7)

$$
\begin{aligned}
f_{A}(v) & =g_{m i} g_{m r} \frac{\left(d_{1}-x\right)^{3}\left(d_{2}+x\right)^{3} S e^{-2 \gamma z}}{\left(w^{2} l_{w}^{2}+z^{2} l_{v}^{2}\right)^{5 / 2}} \\
& =\frac{g_{m t} g_{m r}}{l_{w}{ }^{5}} \frac{\left(d_{1}-x\right)^{3}\left(d_{2}+x\right)^{3} S e^{-2 \gamma z}}{\left(\left(\frac{w}{r}\right)^{2}+z^{2}\right)^{5 / 2}}
\end{aligned}
$$


where $f_{A}(v)$ is $f(v)$ with the effects of anisotropy included. Using the square pattern as was done in this paper, with geometric limits acting as a cutoff for the antenna beamwidths, it will be shown that, at least qualitatively, anisotropy will have some effect when using high gain antennas. As can be seen using (3-18), only the $w$ integration is affected. A typical $w$ integral using the geometric limits for anisotropic turbulence would be of the form

$$
\int_{0}^{\delta\left(d_{2}+x\right)} \overline{\frac{d w}{\left[\left(\frac{w}{r}\right)^{2}+z^{2}\right]^{5 / 2}} .}
$$

Let $\frac{w}{r}=v$. Then (3-19) becomes

$$
r \int_{0}^{\frac{\delta}{r}\left(d_{2}+x\right)} \frac{d v}{\left(v^{2}+z^{2}\right)^{5 / 2}}
$$

This integrand is the same as in the case of isotropic turbulence, but the range of integration is larger, since $r<1$. Thus, the values of $G_{L}$ obtained here would be lower if the effects of anisotropy were included. This effect is the same as if antennas with larger beamwidths in the horizontal dimension than in the vertical dimension were used in an isotropic atmosphere.

\section{Results}

The integrals in the preceding section were calculated for numerous combinations of the parameters. Typical curves which resulted from these calculations are plotted in figure 6 . The curves in figure 6 corresponding to path symmetry $(s=1)$, and identical antennas $(\mu=1)$, are shown again in figure 7 using a different coordinate system which facilitates interpolation. Because of the number of parameters involved it was found impractical to show all of the curves of the type shown in figure 6, and instead, an empirical formula was derived, using these curves, which gives good accuracy using only two graphs and a few simple calculations. The following discussion defines the necessary parameters and shows how to use the empirical curves.

The basic parameters which depend on the path geometry are shown in figure 3 and are as follows:

$$
\alpha_{0}, \quad \beta_{0}, \quad d,
$$

from which may be calculated

$$
\begin{aligned}
& s=\frac{\alpha_{0}}{\beta_{0}}, \\
& \theta=\alpha_{0}+\beta_{0}
\end{aligned}
$$

and

$$
h_{0}=\frac{s}{(1+s)^{2}} d \theta \text {. }
$$

Combining these with the rate of decay of the variance of the refractive index with height, $\gamma$, one obtains

$$
\eta=\gamma d \theta
$$

and

$$
\nu=2 \gamma h_{0}=\frac{2 \eta s}{(1+s)^{2}} .
$$

For the purpose of illustration, ${ }^{2} \gamma=0.23 /$ mile will be used here.

\footnotetext{
${ }^{2}$ For additional values of $\gamma$, see reference [10].
} 


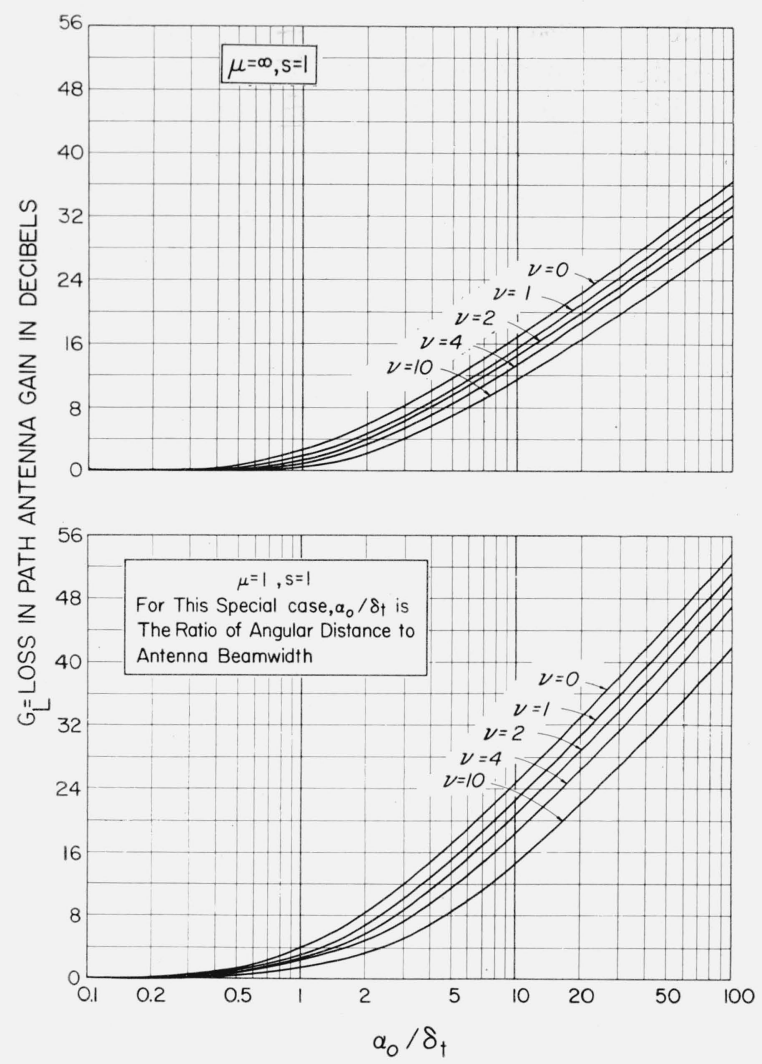

Figure 6. Loss in path antenna gain.

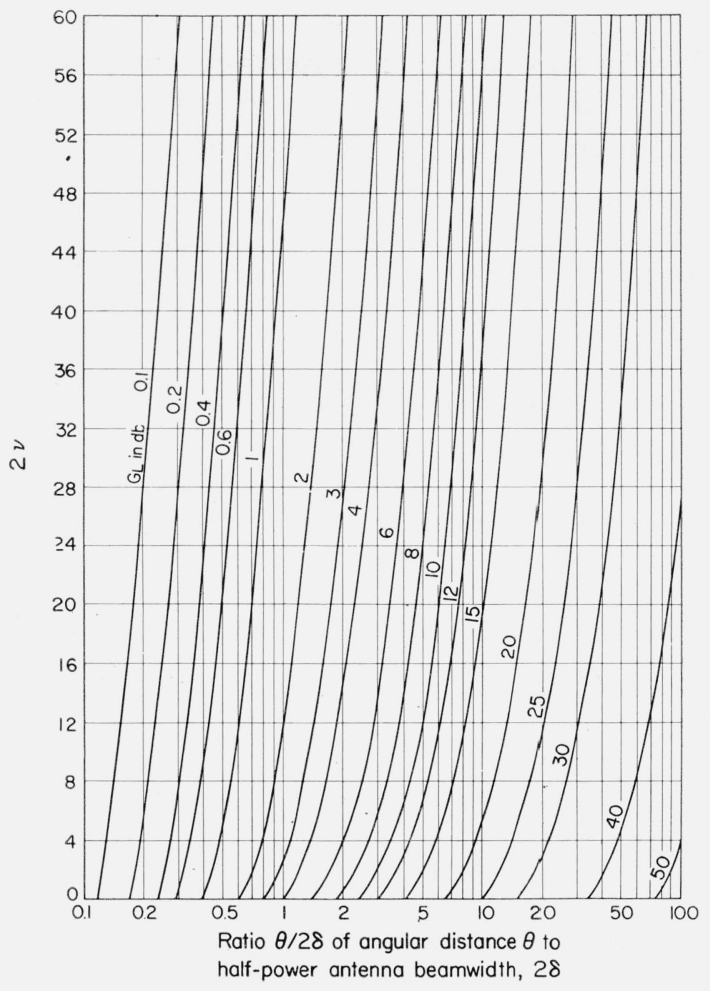

FIGURE 7. Loss in antenna gain, $\mathrm{G}_{\mathrm{L}}$ assuming equal free space gains $\mathrm{G}_{\mathrm{t}}$ and $\mathrm{G}_{\mathrm{r}}$ at the terminals of a symmetrical path $\delta_{\mathrm{t}}=$ $\delta_{\mathrm{r}}=\delta, \mathrm{s}=1$.

The parameters which depend on the antenna characteristics are the half power semibeamwidths, $\delta_{t}$ and $\delta_{r}$. These are used to calculate

$$
\mu=\delta_{r} / \delta_{t}
$$

At the end of this section, formulas are given for calculating $\delta$ from the free space gain of the antenna or from the frequency and area of the antenna aperture.

In the empirical formula, the two parameters $s$ and $\mu$, are combined in the single parameter $s \mu$, and two separate cases arise.

Case $I, s \mu \geq 1$.

Calculate

$$
n=\alpha_{0} / \delta_{t} .
$$

Read $f(\nu)$ from figure 8 and calculate

$$
\hat{n}=(n+0.03 \nu) / f(\nu) \text {. }
$$

Read $G_{L}$ from figure 9 for the appropriate value of $s \mu$. Linear interpolation is possible using the formula

$$
G_{L}=G_{L}^{\prime}+\frac{\frac{1}{s \mu}-\frac{1}{(s \mu)^{\prime}}}{\frac{1}{(s \mu)^{\prime \prime}}-\frac{1}{(s \mu)^{\prime}}}\left(G_{L}{ }^{\prime \prime}-G_{L}{ }^{\prime}\right),
$$




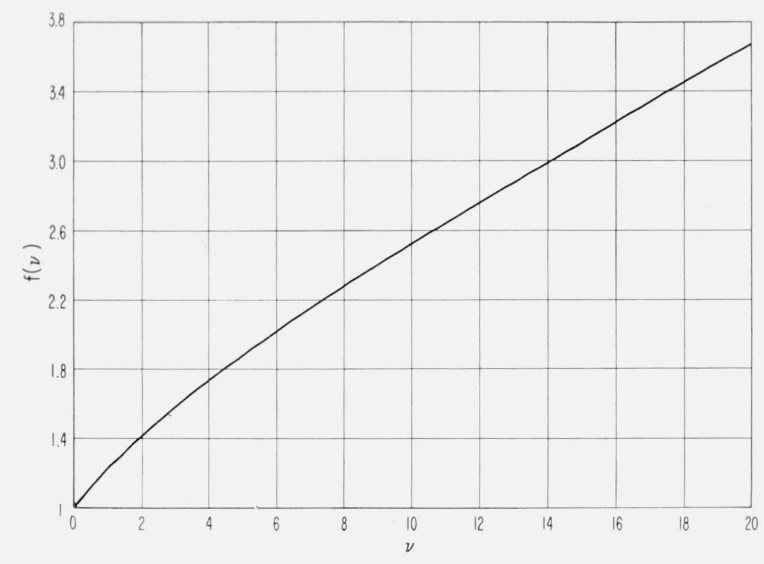

Figure 8. The contraction factor $\mathrm{f}(\nu)$. Supplementary Values.

$\begin{array}{lllll}\nu & f(\nu) & \nu & f(\nu) \\ 1 & 1.22 & 5 & 1.88 \\ 2 & 1.41 & 15 & 3.10\end{array}$

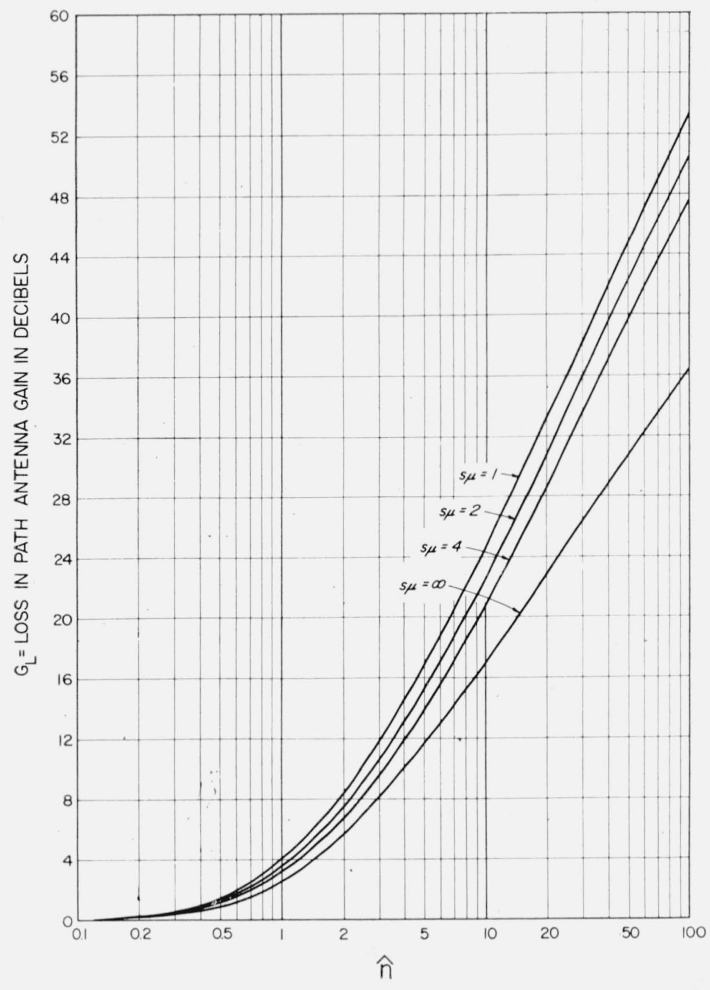

FIGURE 9. Loss in path antenna gain versus $\hat{\mathbf{n}}$ and the product $\mathrm{s} \mu$ for $\nu=0$.

where $G_{L}^{\prime}$ and $G_{L}{ }^{\prime \prime}$ correspond to the graphically obtained values for $(s \mu)^{\prime}$ and $(s \mu)^{\prime \prime}$ less than $s \mu$ and greater than $s \mu$, respectively.

Case $I I, s \mu<1$.

Calculate

$$
n=\beta_{0} / \delta_{r} .
$$

Read $f(\nu)$ from figure 8 and calculate

$$
\hat{n}=(n+0.03 \nu) / f(\nu) .
$$

Read $G_{L}$ from figure 9 on the curve corresponding to the value of $1 / s \mu$. Use formula (4-7) for interpolating replacing $(s \mu)$ by $(1 / s \mu),(s \mu)^{\prime} /$ by $(1 / s \mu)^{\prime}$ and $(s \mu)^{\prime \prime}$ by $(1 / s \mu)^{\prime \prime}$.

If the gain $G$ in decibels is known, the half-power semibeamwidth may be calculated using the following formulas which may be derived from the formulas in section 2 , and are given here for a parabolic dish of diameter $D$ feet at a frequency $f_{m c}$ megacycles per second. $G$ is given by

$$
\begin{aligned}
G & =10 \log _{10}\left[0.56 \pi^{2}(D / \lambda)^{2}\right] \\
& =20 \log _{10} D_{f t}+20 \log _{10} f_{m c}-52.431 \mathrm{db} .
\end{aligned}
$$

Also,

$$
G=10 \log _{10}\left[8.2 /(2 \delta)^{2}\right]
$$

with $\delta$ in radians, and thus,

$$
\delta=1432 \times 10^{-G / 20}=609 \lambda / D \mathrm{mr} .
$$




\section{Comparison with Previous Estimates}

In figure 10, our estimates of $G_{L}$ are plotted together with the estimates given by the previous authors [1, 2, 3]. The values from Staras' [3] paper which are shown in figure 10 correspond to the assumption of isotropic turbulence. For this comparison, it was necessary to use only the curves corresponding to the assumptions of path symmetry and identical antennas on each end of the path since some of the previous estimates are available only for these cases. In addition, our assumption that the gradient of refractivity of the atmosphere decreases exponentially with height leads to the parameter $\nu=2 \gamma h_{\mathrm{o}}$ which does not appear in the other papers. For making this comparison we chose the typical value of $\nu=2$ corresponding to $\gamma \simeq 0.23$, $d \simeq 300 \mathrm{mi}$, and $\theta \simeq 50 \mathrm{mr}$. Our values of $G_{L}$ in figure 10 are lower than the other estimates, and

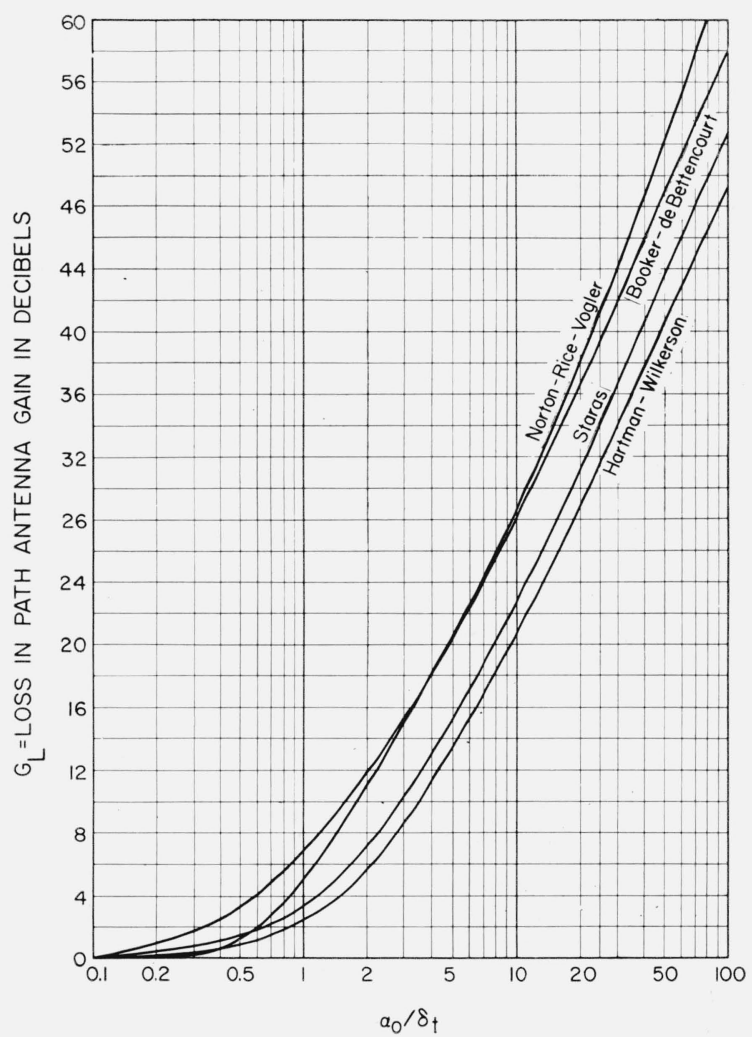

FIGURE 10. A comparison of loss in path antenna gain obtained from different scatter theories for a symmetrical path for $\mathrm{d}=300$ miles, $\mu=\delta_{\mathrm{r}} / \delta_{\mathrm{t}}=1, \mathrm{~s}=\alpha_{\mathrm{o}} / \beta_{\mathrm{o}}=1$ (antennas on the surface).

as can be seen in figure 6 , they would be even lower if a larger value of $\nu$ were used or if an allowance were made for some degree of anisotropy. The difference between our curve and the others is primarily the result of the use of the exponential model atmosphere which is supported by ample data [10] and a scattering cross section that is proportional to $[2 \sin \{(\alpha+\beta) / 2\}]^{-5}$.

The authors acknowledge the assistance of P. L. Rice and H. K. Hansen in the preparation of this paper. 


\section{Appendix 1}

The approximation

$$
\frac{1}{\left[2 \sin \left(\frac{\alpha+\beta}{2}\right)\right]^{5}\left(R R_{0}\right)^{2}} \cong \frac{\left(d_{1}-x\right)^{3}\left(d_{2}+x\right)^{3}}{d^{5}\left(w^{2}+z^{2}\right)^{5 / 2}}
$$

is usually derived by assuming that $\alpha+\beta$ is small [1]. The following method is used to obtain this approximation, because it has the advantage that the error is evaluated. Let

$$
P=\frac{R+R_{0}+d}{2}
$$

Then, using a well known formula from geometry,

$$
\begin{aligned}
{\left[2 \sin \left(\frac{\alpha+\beta}{2}\right)\right]^{-5}=2^{-5} } & \left\{\left(\frac{\left(P-R_{0}\right)(P-d)}{R_{0} d}\right)^{1 / 2}\left(\frac{P\left(P-R_{0}\right)}{R d}\right)^{1 / 2}\right. \\
& \left.+\left(\frac{P(P-R)}{R_{0} d}\right)^{1 / 2}\left(\frac{(P-R)(P-d)}{R d}\right)^{1 / 2}\right\}^{-5}=2^{-5}\left\{\frac{\left(R+R_{0}\right)^{2}-d^{2}}{4 R R_{0}}\right\}^{-5 / 2} .
\end{aligned}
$$

From the geometry

$$
\begin{aligned}
& R_{0}=\sqrt{\left(d_{1}-x\right)^{2}+z^{2}+w^{2}} \\
& R=\sqrt{\left(d_{2}+x\right)^{2}+z^{2}+w^{2}}
\end{aligned}
$$

and

$$
R R_{0}=\left(d_{1}-x\right)\left(d_{2}+x\right)\left\{1+\frac{z^{2}+w^{2}}{\left(d_{1}-x\right)^{2}}\right\}^{1 / 2}\left\{1+\frac{z^{2}+w^{2}}{\left(d_{2}+x\right)^{2}}\right\}^{1 / 2}
$$

(6-5) can be expanded if $z^{2}+w^{2}<\left(d_{1}-x\right)^{2}$ and $z^{2}+w^{2}<\left(d_{2}+x\right)^{2}$. This is generally the case for a beyond-the-horizon propagation path. Then,

$$
R R_{0}=\left(d_{1}-x\right)\left(d_{2}+x\right)+\frac{z^{2}+w^{2}}{2}\left\{\frac{\left(d_{2}+x\right)^{2}+\left(d_{1}-x\right)^{2}}{\left(d_{1}-x\right)\left(d_{2}+x\right)}\right\}+\cdots
$$

Using (6-3), (6-4), and the first two terms of (6-6), we obtain

$$
\left(R+R_{0}\right)^{2}-d^{2} \simeq 2\left(z^{2}+w^{2}\right)\left\{1+\frac{\left(d_{2}+x\right)^{2}+\left(d_{1}-x\right)^{2}}{2\left(d_{1}-x\right)\left(d_{2}+x\right)}\right\}=\frac{\left(z^{2}+w^{2}\right) d^{2}}{\left(d_{1}-x\right)\left(d_{2}+x\right)} .
$$

Using (6-2), (6-7), and the first term of (6-6) we obtain

$$
\frac{\left[2 \sin \left(\frac{\alpha+\beta}{2}\right)\right]^{-5}}{\left(R R_{0}\right)^{2}}=\frac{\left(R R_{0}\right)^{1 / 2}}{\left[\left(R+R_{0}\right)^{2}-d^{2}\right]^{5 / 2}} \simeq \frac{\left(d_{1}-x\right)^{3}\left(d_{2}+x\right)^{3}}{d^{5}\left(w^{2}+z^{2}\right)^{5 / 2}},
$$

which is the same as $(3-6)$. 


\section{Appendix 2}

The derivation of $(3-15)$ with $g_{m t}=1, g_{m r}=1$ is as follows:

$$
\begin{aligned}
K(s, \eta, 2,2)=\frac{3 \lambda S}{\pi d^{3}}\left\{\int_{0}^{d_{1}} d x \int_{z_{1 m}}^{\infty} d z \int_{-\infty}^{\infty} d w \frac{\left(d_{1}-x\right)^{3}\left(d_{2}+x\right)^{3} e^{-2 \gamma z}}{\left(w^{2}+z^{2}\right)^{5 / 2}}\right. \\
\left.\quad+\int_{-d_{2}}^{0} d x \int_{z_{2 m}}^{\infty} d z \int_{-\infty}^{\infty} d w \frac{\left(d_{1}-x\right)^{3}\left(d_{2}+x\right)^{3} e^{-2 \gamma z}}{\left(w^{2}+z^{2}\right)^{5 / 2}}\right\}=K_{1}+K_{2},
\end{aligned}
$$

where $K_{1}$ and $K_{2}$ correspond to the scattering regions to the left and right of the crossover $C$ of radio horizon rays in figure 3 .

$$
\begin{aligned}
& z_{1 m}=\beta_{0}\left(d_{2}+x\right) . \\
& z_{2 m}=\alpha_{0}\left(d_{1}-x\right) .
\end{aligned}
$$

In $K_{1}$, let

$$
x=k d_{1}
$$

and

$$
z_{0}=\frac{\alpha_{0} d_{1}}{z}\left(1+\frac{k}{s}\right)
$$

where

In $K_{2}$ let

$$
s=\alpha_{0} / \beta_{0}=d_{2} / d_{1}
$$

$$
x=-k d_{2}
$$

and

$$
z_{0}=\frac{\beta_{0} d_{2}}{z}(1+s k)
$$

Then, integrating with respect to $w$, we obtain

$$
\begin{aligned}
K_{0}=\frac{4 \lambda S d}{\pi \theta^{3}(1+s)}\left\{\int_{0}^{1}(1-k)^{3} d k \int_{0}^{1} z_{0}^{2} \exp \left[\frac{-\nu}{z_{0}}(1+k / s)\right] d z_{0}\right. \\
\left.\quad+s \int_{0}^{1}(1-k)^{3} d k \int_{0}^{1} z_{0}^{2} \exp \left[\frac{-\nu}{z_{0}}(1+s k)\right] d z_{0}\right\},
\end{aligned}
$$

where we have set

$$
\nu=2 \gamma h_{0}=2 \gamma \alpha_{0} d_{1}=2 \gamma \beta_{0} d_{2} .
$$

Let

$$
L_{1}=\frac{1}{1+s} \int_{0}^{1}(1-k)^{3} d k \int_{0}^{1} z_{0}^{2} \exp \left[\frac{-\nu}{z_{0}}(1+k / s)\right] d z_{0}
$$

and

$$
L_{2}=\frac{s}{1+s} \int_{0}^{1}(1-k)^{3} d k \int_{0}^{1} z_{0}^{2} \exp \left[\frac{-\nu}{z_{0}}(1+s k)\right] d z_{0}
$$

We need only consider $(7-7 \mathrm{a})$ since $(7-7 \mathrm{~b})$ can be obtained from $(7-7 \mathrm{a})$ by replacing $s$ by $1 / s$.

Thus, performing the integration with respect to $k$, we obtain:

$$
L_{1}=\frac{1}{1+s} \int_{0}^{1} z_{0}^{2} \exp \left(-\nu / z_{0}\right)\left\{\frac{s z_{0}}{\nu}-\frac{3\left(s z_{0}\right)^{2}}{\nu^{2}}+\frac{6\left(s z_{0}\right)^{3}}{\nu^{3}}-\frac{6\left(s z_{0}\right)^{4}}{(\nu)^{4}}+\frac{6\left(s z_{0}\right)^{4} \exp \left[-\nu /\left(s z_{0}\right)\right]}{\nu^{4}}\right\} d z_{0} .
$$


Let

and

$$
\begin{aligned}
& \frac{\nu}{z_{0}}=u, \\
& \nu_{2}=\nu(1+1 / s),
\end{aligned}
$$

Then

$$
c_{1}=\frac{s^{4}+7 s^{3}+21 s^{2}+35 s}{5}
$$

$$
\begin{aligned}
L_{1}=\frac{\nu^{3}}{1+s} \int_{\nu}^{\infty}\left\{e^{-u}\left[\frac{s}{u^{5}}-\frac{3 s^{2}}{u^{6}}+\frac{6 s^{3}}{u^{7}}-\frac{6 s^{4}}{u^{8}}\right]+\frac{6 s^{4} e^{-u\left(1+\frac{1}{s}\right)}}{u^{8}}\right\} d u \\
=\frac{\nu^{3}}{7(1+s)}\left\{e^{-\nu}\left[-\frac{6 s^{4}}{\nu^{7}}+\frac{s^{4}+7 s^{3}}{\nu^{6}}-\frac{s^{4}+7 s^{3}+21 s^{2}}{5 \nu^{5}}+\frac{c_{1}}{24}\left(\frac{6}{\nu^{4}}-\frac{2}{\nu^{3}}+\frac{1}{\nu^{2}}-\frac{1}{\nu}\right)\right]-\frac{c_{1}}{24} \operatorname{Ei}(-\nu)\right. \\
\left.+6 s^{4}(1+1 / s)^{7}\left[\frac{1}{\nu_{2}^{7}}-\frac{1}{6 \nu_{2}^{6}}+\frac{1}{30 \nu_{2}^{5}}-\frac{1}{120 \nu_{2}^{4}}+\frac{1}{360 \nu_{2}^{3}}-\frac{1}{720 \nu_{2}^{2}}+\frac{1}{720 \nu_{2}}+\frac{1}{720} \operatorname{Ei}\left(-\nu_{2}\right)\right]\right\} .
\end{aligned}
$$

where

$$
-E i(-x)=\int_{x}^{\infty} \frac{e^{-t}}{t} d t
$$

Thus, adding $L_{1}$ and $L_{2}$ we obtain:

$$
\begin{array}{r}
K_{0}=\frac{\lambda S d}{210 \pi \theta^{3} \nu^{4}(s+1) s^{3}}\left\{e ^ { - \nu } \left[-720\left(s^{7}+1\right)+120\left(s^{7}+7 s^{6}+7 s+1\right) \nu-24 \nu^{2}\left[(s+1)^{7}\right.\right.\right. \\
\left.\left.-35 s^{3}(s+1)\right]+(s+1)^{7} \nu^{3}\left(6-2 \nu+\nu^{2}-\nu^{3}\right)\right]-(s+1)^{7} \nu^{7}[\operatorname{Ei}(-\nu)-E i(-\nu[1+1 / s]) \\
-E i(-\nu[1+s])]+s e^{-\nu(1+1 / s)}\left[720 s^{6}-120 s^{5}(1+s) \nu+24 s^{4}(1+s)^{2} \nu^{2}-6 s^{3}(1+s)^{3} \nu^{3}\right. \\
\left.+2 s^{2}(1+s)^{4} \nu^{4}-s(1+s)^{5} \nu^{5}+(1+s)^{6} \nu^{6}\right]+e^{-\nu(1+s)}\left[720-120(1+s) \nu+24(1+s)^{2} \nu^{2}\right. \\
\left.\left.\quad-6(1+s)^{3} \nu^{3}+2(1+s)^{4} \nu^{4}-(1+s)^{5} \nu^{5}+(1+s)^{6} \nu^{6}\right]\right\} .
\end{array}
$$

Figure 11 shows $3 \pi \theta^{3}(1+s) K_{0} / 2 \lambda S d$ plotted versus $\eta / 2$ for several values of the path asymmetry factor, $s$.

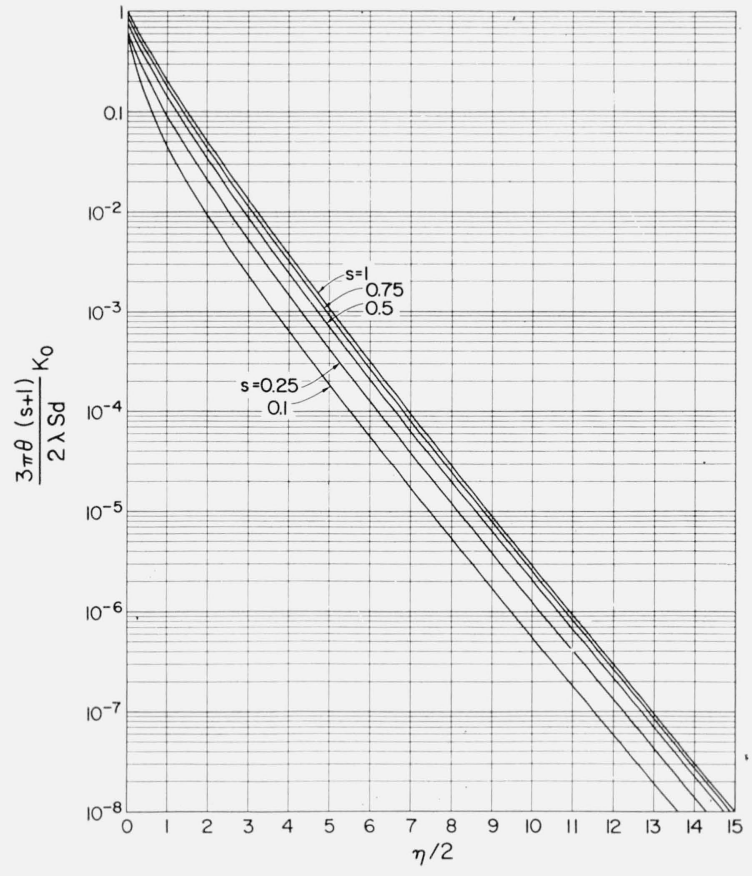

Figure 11. Dependence of the scattering integral on $\mathrm{s}$ and $\eta$. 


\section{References}

[1] K. A. Norton, P. L. Rice, and L. E. Vogler, The use of angular distance in estimating transmission loss and fading range for propagation through a turbulent atmosphere over irregular terrain, Proc. IRE 43, 1428, (1955).

[2] H. G. Booker and J. T. de Bettencourt, Theory of radio transmission by tropospheric scattering using very narrow beams, Proc. IRE 43, 281, (1955).

[3] Harold Staras, Antenna-to-medium coupling loss, PGAP AP-5, 288, (1957).

[4] Samuel Silver, Microwave antenna theory and design, pp. 169-199 (McGraw-Hill, 1949).

[5] Reference data for radio engineers, International Telephone and Telegraph Corp., p. 703, 4th ed.

[6] K. A. Norton, Point-to-point relaying via the scatter mode of tropospheric propagation, Trans. IRE, Prof. Gp. on Comm. Systems CS-4, No. 1, 39, (1956).

[7] H. G. Booker and W. E. Gordon, The role of stratospheric scattering in radio communication, E. E. Report (Cornell University, Ithaca, New York, 1956).

[8] R. L. Abbott (private communication).

[9] H. Staras, Forward scattering of radio waves by anisotropic turbulence, Proc. IRE 43, 1374 (1955).

[10] B. R. Bean and G. D. Thayer, Models of atmospheric radio refractive index, to be published in Proc. IRE 47, 5, (1959). (Final pages to be set in proof.)

Boulder, Colo.

(Paper 63D3-23) 